Soukas, Alexander

\section{Gene expression profile induced by lep- tin in white adipose tissue and liver}

\author{
Alexander Soukas, Paul Cohen \& J effrey M. Friedman \\ Laboratory of M olecular Genetics, Rockefeller University, \\ New York, New York 10021, USA
}

Leptin, the product of the ob gene, is a hormone that regulates body weight by decreasing food intake and increasing energy expenditure. After binding to its receptor in the hypothalamus and other tissues, leptin induces a novel metabolic response, which is physiologically distinct from food restriction alone. To better characterise this metabolic response, we monitored gene expression in lean and obese mice after leptin treatment or pair-feeding (food restriction) using Affymetrix GeneChips. We report the specific gene expression profile induced by leptin in white adipose tissue and liver. From these analyses, it is possible, for the first time, to distinguish leptin treatment from food restriction on a molecular level, as a number of genes are differentially expressed between the two states in the aforementioned tissues. This information will make it possible to further characterise leptin's novel metabolic effects and the efferent signals that coordinate them.

\section{Spellman, Paul}

\section{Functional clustering of genes using microarray gene expression data}

\section{Paul Spellman', Audrey Gasch², M ichael Eisen ${ }^{1}$, Camilla Kao², $^{2}$ Patrick Brown ${ }^{2,3} \&$ David Botstein ${ }^{1}$}

Departments of ${ }^{1}$ Genetics, ${ }^{2}$ Biochemistry and ${ }^{3}$ How ard Hughes M edical Institute, Stanford University M edical School, Palo Alto, California 94305, USA

Gene expression data generated using microarrays can be used to group genes into clusters that reflect their involvement in biological processes ${ }^{1}$. We have more than tripled the gene expression data available to include approximately 25 physiologically different conditions assayed with approximately 400 individual array hybridizations (each hybridization measures mRNA levels for each gene in the entire genome). This data set now contains characterizations of gene expression changes in response to numerous stresses, as well as changes during the cell cycle, sporulation and nutritional conditions. Such a large data set $(\sim 2.5$ million independent observations) permits only a cursory description in words; instead, these data are the foundation for a reference physiological gene expression database. An example of the kinds of information one can extract from these data is the genome-wide pattern of gene expression in response to stress. In addition to generic negative responses (for example, turning down transcription of the genes responsible for translation or progress through the cell cycle), there are a large number of genes that are induced by virtually any stress. There are far fewer genes whose induction is seen only in a particular type of stress. Of particular interest is the overlap in gene expression between oxidative stress and heat shock. We hope that this data set will be useful in providing a context for a great variety of physiological measurements in the future.

1. Eisen, M.B. et al. Proc. Natl Acad. Sci. USA 95, 14863-14868 (1998).
Statham, Victoria

\section{Applying cDNA microarray technology to gene therapy}

\author{
Victoria Statham, Michael Bittner, J effrey Trent \\ \& Richard A. M organ
}

Clinical Gene Therapy Branch, Cancer Genetics Branch, National Human

Genome Research Institute, NIH, Bethesda, M aryland, USA

Graduate Genetics Program, The George Washington University,

Washington DC, USA

Gene therapy has often been described as a "black box" technique; that is, it is not known what happens to the cell after vector-mediated gene transfer. Although expression levels of the transgene are monitored, very little is known about how cellular gene expression is affected by the transduction process or transgene expression. Perturbations of cellular gene expression may be deleterious, even harmful, in several ways and could decrease the efficacy of therapy by altering the expression of the therapeutic gene. Studying the global changes in gene expression of cells transduced with different viral vectors is necessary for understanding the biology of gene therapy, and should yield safer, more effective therapies. To analyse these potential changes in gene expression more comprehensively, we are using the recently described cDNA microarray to look at cells transduced with a neoR-expressing retroviral vector. The cDNA microarray consists of numerous cDNAs representing genes and ESTs spotted individually on a microscope slide. Total RNA from transduced and untransduced cell populations are reverse transcribed with a fluorescently labelled nucleotide to produce differently labelled cDNA probes. These probes are mixed and hybridized to the cDNA targets on the microarray, which is then scanned to record fluorescence intensities for each probe at each target. A ratio of these intensities is used to identify expression differences between the cell populations, and signal intensity can approximate RNA quantity. This technique using the $1.4 \mathrm{~K}$ array allows us to look at the expression of more than 1,200 host genes at one time, and in so doing, screen for alterations caused by transduction or the expression of this commonly used bacterial reporter gene. Preliminary data indicate that the expression of several genes is significantly altered when cells are analysed immediately after selection. When these same cell populations are removed from growth in G418-containing selection medium and cultured for several months, significant changes in gene expression were not observed. These studies will help to address potential safety concerns as well as provide information to be used as a basis for designing better vectors.

Steffen, Martin

\section{Surface attachment chemistry for in situ probe DNA amplification}

\author{
Martin Steffen, Rob Mitra, Xiaohua Huang, John Aach \\ \& George Church
}

Dept. of Genetics, Harvard M edical School, Boston, M assachusetts, USA

Routine expression analysis of complete mammalian genomes will require the extension of several existing technologies. Spotters will need to be able to print features more densely, and the cost and labor involved in preparing probe DNA for slide manufacture needs to be reduced. To meet these goals, we employ noncontact piezoelectric ink-jet print heads. These print heads can produce features as small as 50 microns, and we routinely spot samples at densities allowing 100,000 features per slide. With 75 micron center-to-center spacing, one would be able to spot 200,000 features per slide. Another advantage of ink-jet print heads is that features can be printed without stopping spotter movement, and can therefore achieve printing speeds of 20 spots per second per printing head. DNA prepara- 